\title{
Tigecycline Induced Fibrinogen Decrease in Critically III Patients with Multidrug Resistant Bacterial Infections - an Observational Retrospective Study
}

Junfeng Heng

The Affiliated Wuxi People's Hospital of Nanjing Medical University

Xiuhong Zhang

The Affiliated Wuxi People's Hospital of Nanjing Medical University

\section{Fengming Liang}

The Affiliated Wuxi People's Hospital of Nanjing Medical University

\section{Qiuhui Wang}

The Affiliated Wuxi People's Hospital of Nanjing Medical University

\section{Hongyang $X u$}

The Affiliated Wuxi People's Hospital of Nanjing Medical University Jiaojie Hui ( $\square$ huijiaojie15@163.com )

The Affiliated Wuxi People's Hospital of Nanjing Medical University

\section{Research Article}

Keywords: tigecycline, fibrinogen decrease, retrospective

Posted Date: January 18th, 2022

DOI: https://doi.org/10.21203/rs.3.rs-1245134/v1

License: () (1) This work is licensed under a Creative Commons Attribution 4.0 International License. Read Full License 


\begin{abstract}
A decrease of plasma fibrinogen level has occasionally been observed during tigecycline treatment. The present observational retrospective study was performed to analyze the effect of tigecycline on coagulation function in critically ill patients with multidrug-resistant bacterial infections, especially on plasma fibrinogen level. 83 adult subjects treating with tigecycline were enrolled. Coagulation function spanning all three time periods (berore, during and after tigecycline administration) was determined to measure the effects of tigecycline. A striking decrease of fibrinogen was noted during tigecycline treatment $(P=0.000)$, and this change started to reverse when tigecycline was stopped. In the higher-dose group, fibrinogen also dropped significantly during tigecycline treatment $(P=0.009)$, and after the cessation of tigecycline, the fibrinogen level did not rebound, but kept on drop. The fibrinogen levels dropped significantly in the 4 patients developed new-onset bleeding during tigecycline treatment, showing almost simultaneously. There were increased prothrombin time (PT), activated partial thromboplastin time (APTT), thrombin time (TT), platelet count (PLT) and decreased platelet level during tigecycline treatment and a reversal when tigecycline was stopped, although only the difference of PT reached significance $(P=0.043)$. Tigecycline induced fibrinogen decrease in critically ill patients with multidrug-resistant bacterial infections, and this change started to reverse when tigecycline was stopped.
\end{abstract}

\title{
Introduction
}

With the increasing emergence of multidrug-resistant organisms in critically ill patients, therapeutic options have become limited, especially when resistance develops to previously susceptible organisms [1-5]. Tigecycline is a broad-spectrum antibiotic indicated for the treatment of complicated intra-abdominal infections and complicated skin and skin-structure infections as well as community-acquired bacterial pneumonia in adults [3]. Tigecycline offers potential advantages over other parenterally administered antimicrobial agents because of its expanded spectrum of coverage against gram-positive, anaerobic, gramnegative, and multiply antimicrobial resistant microorganisms [6].

The manufacturer provided information that tigecycline might affect the coagulation function, although an uncommon adverse reaction (incidence < $2 \%$ ) [7]. Coagulation disorder, such as prolongation of APTT (activated partial thromboplastin time) and PT (prothrombin time), increase of international normalized ratio (INR), and decrease of blood platelets, has indeed been observed during tigecycline treatment [8-12]. Additionally, a decrease of plasma fibrinogen level has occasionally been observed during tigecycline treatment, and when discontinued the drug, the fibrinogen improved markedly [13-18], giving first place to case report. The strong time-dependent association of fibrinogen and the use of tigecycline and its reversal after discontinuation might point to a causative role of the drug.

Tigecycline is usually used to treat severe multidrug-resistant infection in critically ill patients, who are at higher risk of coagulation disorder even bleeding. Therefore, the present observational retrospective study was performed to analyze the effect of tigecycline on coagulation function in critically ill patients with multidrug-resistant bacterial infections especially on plasma fibrinogen level.

\section{Methods}

\section{Study population}

This observational retrospective study was conducted in The Affiliated Wuxi People's Hospital of Nanjing Medical University, a general grade-A hospital of China, with 1800 bed capacity. 83 adult subjects ( 24 in general intensive care unit, 21 in thoracic surgery intensive care unit, 15 in hematology department, 11 in respiratory intensive care unit, 5 in cerebral surgery department, 3 in invasive technology department, 3 in coronary care unit, 3 in neurology intensive care unit, and 1 in gastroenterology department) were enrolled treating with tigecycline between 1st January 2018 and 31 st May 2020.

\section{Study design}

Tigecycline was administered at a dose of $50 \mathrm{mg}$ i.v. every $12 \mathrm{~h}$ after a loading dose of $100 \mathrm{mg}$ usually. There were also 18 patients with high-dose tigecycline treatment (100 mg i.v. $12 \mathrm{~h}$, with a $200 \mathrm{mg}$ loading dose). Exclusion criteria were: age $<18$ years, duration of treatment with tigecycline for $<5$ days, coagulation function might be significantly affected (extracorporeal membrane oxygenation, continuous renal replacement therapy, or serious blood disease), with bleeding manifestations, receiving blood transfusion or supplement of fibrinogen to ameliorate coagulation disorder after tigecycline treatment. As an observational retrospective study, the protocol was approved by Ethics Committee of the Affiliated Wuxi People's Hospital of Nanjing Medical University and we received the agreement that it waived the need for informed consent. All methods were performed conform to CONSORT 2010 guidelines.

\section{Observation indexes}

Samples with coagulation function monitoring spanning all three time periods (berore, during and after tigecycline administration) were taken into statistical analysis. Fibrinogen (FIB) levels, PT, APTT, thrombin time (TT), platelet (PLT) count, alanine aminotransferase (ALT) levels, total bilirubin (TB) levels, and creatinine $(\mathrm{Cr})$ levels were determined to measure the effects of tigecycline.

\section{Statistical analysis}

Data were analyzed using SPSS software, version 13.0 (SPSS Inc., Chicago). 95\% confidence interval (95\% CI) was calculated. ANOVA was applied by the Bonferroni testing as post hoc analysis for examination of coagulation function(FIB, PT, APTT and TT), PLT count, Liver function (ALT and TB), kidney function(Cr)at different time point. The results were considered statistically significant if $P<0.05$. Pearson correlations between FIB and ALT, TB, Cr were performed.

\section{Results}




\section{Characteristics of included subjects}

Among the 83 subjects treated with tigecycline for multi-drug resistant bacterial infections, 73 were treated for ventilator-associated and healthcare-acquired pneumonia, 4 biliary tract infections, 3 intra-abdominal infections, and 3 undefined sever infections. The maximum species of pathogens was multi-drug resistant Acinetobacter baumannii, with a percentage of 63.9\% (53/83), and other pathogens were gram-negative bacteria (Escherichia coli (E. coli), Enterobacter cloacae, Klebsiell pneumoniae, Acinetobacter spp, Pseudomonas aeruginosa, and Stenotrophomonas maltophilia.

50 subjects were excluded from further analyzed for received consecutively treatment with tigecycline for $<5$ days, or coagulation function might be significantly affected (extracorporeal membrane oxygenation, continuous renal replacement therapy or serious blood disease), or with bleeding manifestations, or receiving blood transfusion and supplement of fibrinogen to ameliorate coagulation disorder after tigecycline treatment. The number of subjects taken into statistical analysis with above exclusion criteria and coagulation function monitoring spanning all three time periods (berorelduring and after tigecycline administration) was 33 , with a percentage of $39.8 \%$ (33/83). The mean age was $61.3 \pm 20.5$ years (from 21 to 90 years). There were 27 male and 6 female subjects.

\section{Decrease of fibrinogen during tigecycline treatment}

A striking decrease of fibrinogen was noted during tigecycline treatment [from 3.57 (95\% Cl: 3.26-3.88) g/L to 2.34 (95\% Cl: 2.11-2.57) g/L, $P=0.000$; normal range (NR): $2.0-4.0 \mathrm{~g} / \mathrm{L}$ ]. This change started to reverse when tigecycline was stopped [from 2.34 to 2.52 (95\% Cl: 2.02-2.83) g/L)] (Table 1). We did not find significant correlations between fibrinogen and liver function ( $A L T$ : $r=0.048, P=0.600 ; T B: r=0.045, P=0.629$ ) or kidney function (Cr: $r=0.030$, $P=0.736$ ).

Table 1

Differences of biochemical indexes at different time of tigecycline treatment

\begin{tabular}{|c|c|c|c|c|c|c|}
\hline & \multirow{2}{*}{$\begin{array}{l}\text { Pre-tigecycline } \\
(95 \% \mathrm{Cl})(\mathrm{A})\end{array}$} & \multirow{2}{*}{$\begin{array}{l}\text { During-tigecycline } \\
(95 \% \mathrm{Cl})(\mathrm{B})\end{array}$} & \multirow{2}{*}{$\begin{array}{l}\text { Post-Tigecycline } \\
(95 \% \mathrm{Cl})(\mathrm{C})\end{array}$} & \multicolumn{3}{|l|}{$P$ value } \\
\hline & & & & A VS. B & A VS. C & B VS. C \\
\hline \multicolumn{7}{|c|}{ Coagulation function } \\
\hline $\mathrm{FIB}(\mathrm{g} / \mathrm{L})$ & $3.57(3.26-3.88)$ & $2.34(2.11-2.57)$ & $2.52(2.02-2.83)$ & 0.000 & 0.000 & 0.659 \\
\hline $\mathrm{PT}(\mathrm{s})$ & $13.25(11.30-15.20)$ & 15.93(14.22-17.64) & $13.29(9.55-17.03)$ & 0.043 & 0.985 & 0.207 \\
\hline APTT(s) & $39.95(34.29-45.61)$ & $45.87(40.91-50.84)$ & $43.60(32.74-54.46)$ & 0.123 & 0.558 & 0.708 \\
\hline $\mathrm{TT}(\mathrm{s})$ & $29.41(22.99-35.82)$ & $30.21(24.59-35.83)$ & $28.21(15.90-40.52)$ & 0.853 & 0.865 & 0.771 \\
\hline PLT count $\left(* 10^{9} / \mathrm{L}\right)$ & $155.89(131.21-178.56)$ & 136.13(115.36-156.90) & $147.00(101.55-192.45)$ & 0.242 & 0.762 & 0.668 \\
\hline \multicolumn{7}{|l|}{ Liver function } \\
\hline $\operatorname{ALT}(\mathrm{U} / \mathrm{L})$ & $33.06(22.42-43.71)$ & $26.60(19.59-33.60)$ & 33.52(19.71-47.34) & 0.317 & 0.958 & 0.377 \\
\hline TB(umol/L) & $46.35(26.72-65.97)$ & $36.86(23.96-49.77)$ & $31.59(6.12-57.07)$ & 0.426 & 0.365 & 0.716 \\
\hline \multicolumn{7}{|l|}{ kidney function } \\
\hline $\mathrm{Cr}$ (umol/L) & $84.66(59.60-109.71)$ & $94.71(78.24-111.19)$ & $125.43(92.92-157.94)$ & 0.508 & 0.052 & 0.098 \\
\hline
\end{tabular}

The suggested first dose of tigecycline is $100 \mathrm{mg}$ (also for renal failure patients requiring dialysis and Child-Pugh class A and B hepatic dysfunction patients), followed by $50 \mathrm{mg} \mathrm{q12h}$. For Child-Pugh class $\mathrm{C}$ patients, the dose is decreased to $25 \mathrm{mg} \mathrm{q} 12 \mathrm{~h}$. Among the 83 subjects taken into analysis, 65 subjects were administered the suggested dose, and 18 subjects received a higher dose. The fibrinogen level dropped significantly during tigecycline injection in the suggested-dose group, from 3.51 (95\% Cl: 3.17-3.84) g/L to 2.31 (95\% Cl: 2.06-2.56) g/L ( $P=0.000)$ (Figure 1). After the cessation of tigecycline, the fibrinogen level rebounded to 2.46 (95\% Cl: 1.61-3.31) g/L ( $P=0.742)$ (Figure 1). In the higher-dose group, the fibrinogen level also dropped significantly during tigecycline injection, from 3.84 (95\% Cl: 3.01-4.69) g/L to 2.45 (95\% Cl: 1.87-3.04) g/L ( $P=0.009)$ (Figure 2). However, after the cessation of treatment, the fibrinogen level did not rebound, but kept on drop to 1.87 (95\% Cl: 0.78-2.96) g/L ( $P=0.345$ VS. during-tigecycline group, $P=0.000$ VS. pre-tigecycline group) (Figure 2 ).

\section{Characteristics and treatment information of 4 patients developed new-onset bleeding}

Among the 65 subjects in the suggested dose group, 3 (4.6\%) developed new-onset bleeding, and among the 18 subjects in the higher-dose group, 1 (5.5\%) developed new-onset bleeding. The fibrinogen levels dropped significantly in these 4 bleeding subjects, 3 of them with a decline of fibrinogen from normal range to a very slow level in about 1 week, 1 in about 2 weeks, and the new-onset bleeding and fibrinogen drop showed almost simultaneously (Table 2). 
Table 2

Characteristics and treatment information of 4 patients developed new-onset bleeding during tigecycline treat

\begin{tabular}{|c|c|c|c|c|c|c|c|c|c|}
\hline Case & $\begin{array}{l}\text { Infection } \\
\text { site }\end{array}$ & Pathogens & Comorbidity & Concomitant drugs & $\begin{array}{l}\text { Renal failure } \\
\text { (undergoing } \\
\text { hemodialysis) }\end{array}$ & $\begin{array}{l}\text { Hepatic } \\
\text { Impairment }\end{array}$ & $\begin{array}{l}\text { Dosage } \\
(\mathrm{mg})\end{array}$ & $\begin{array}{l}\text { Hemorrhage } \\
\text { Site (days } \\
\text { after } \\
\text { tigecycline) }\end{array}$ & $\begin{array}{l}\text { Fibri } \\
\text { level } \\
\text { near } \\
\text { tigec } \\
\text { (g/L } \\
\text { befo } \\
\text { tigec }\end{array}$ \\
\hline 1 & Pneumonia & Negative & $\begin{array}{l}\text { Diabetes } \\
\text { Hypertension }\end{array}$ & $\begin{array}{l}\text { Cefoperazone- } \\
\text { sulbactam/ } \\
\text { linezolid }\end{array}$ & Yes (No) & No & $\begin{array}{l}50 \\
q 12 h\end{array}$ & $\begin{array}{l}\text { alimentary } \\
\text { tract (5) }\end{array}$ & 2.64 \\
\hline 2 & Pneumonia & Negative & $\begin{array}{l}\text { Multiple } \\
\text { myeloma }\end{array}$ & $\begin{array}{l}\text { Cefoperazone- } \\
\text { sulbactam }\end{array}$ & Yes (No) & No & $\begin{array}{l}50 \\
q 12 h\end{array}$ & urethra (7) & 2.91 \\
\hline 3 & Pneumonia & $\begin{array}{l}\text { Burkholderia } \\
\text { cepacia }\end{array}$ & $\begin{array}{l}\text { After lung } \\
\text { transplantation }\end{array}$ & $\begin{array}{l}\text { Meropenem/ Voriconazole/ } \\
\text { Amphotericin B/Ganciclovir }\end{array}$ & No & No & $\begin{array}{l}50 \\
q 12 h\end{array}$ & airway (3) & 2.75 \\
\hline 4 & Pneumonia & $\begin{array}{l}\text { A. } \\
\text { baumannii }\end{array}$ & Hypertension & Imipenemcilastatin/Alprostadil & No & No & $\begin{array}{l}100 \\
q 12 h\end{array}$ & airway (5) & 4.70 \\
\hline
\end{tabular}

\section{Changes of other observation indexes during tigecycline treatment}

There were increased PT [from 13.25 (95\% Cl: 11.30-15.20) s to 15.93 (95\% Cl: 14.22-17.64) s, $P=0.043$; NR: 9.0-14.5s] $\mathrm{APTT}$ [from 39.95 (95\% Cl: 34.29$45.61)$ s to 45.87 (95\% Cl: $40.91-50.84)$ s, $P=0.123$; NR: $24.0-36.0$ s] $\square T T$ [from 29.41 (95\% Cl: 22.99-35.82) s to 30.21 (95\% Cl: $24.59-35.83$ ) s, $P=0.853$; NR: 15.8-24.9s] and decreased platelet level [from 155.89 (95\% Cl: $131.21-178.56) * 10^{9} / \mathrm{L}$ to $136.13(95 \% \mathrm{Cl}: 115.36-156.90) * 10^{9} / \mathrm{L}, P=0.242 ; \mathrm{NR}: 80-300 * 10^{9} / \mathrm{L}$ ] during tigecycline treatment and a reversal when tigecycline was stopped [15.93s to 13.29 (95\% Cl: 9.55-17.03) s for PT, 45.87s to 43.60 (95\% Cl: $32.74-54.46$ ) s for APTT, 30.21 s to 28.21 (95\% Cl: $15.90-40.52)$ s for TT, $136.13^{*} 10^{9} / \mathrm{L}$ to $147.00(95 \% \mathrm{Cl}: 101.55-192.45) * 10^{9} / \mathrm{L}$ for platelet count], although only the difference of PT before and after tigecycline reached significance (Table 1). There were no significant changes in liver (ALT and TB) and kidney (Cr) function associated with treatment (Table 1).

\section{Discussion}

Tigecycline was approved in 2005 by the US Food and Drug Administration (FDA) and since then has been used as a last-resort treatment option against severe infections caused by multidrug-resistant (MDR) and extensively drug-resistant (XDR) Acinetobacter spp [19]. The combination of tigecycline with another antimicrobial agent is considered even in the treatment of nosocomial pneumonia due to pandrug-resistant Acinetobacter baumannii infections [20]. In our retrospective analysis across 18 months, the maximum species of pathogens of these severe infected patients using tigecycline was multi-drug resistant Acinetobacter baumannii, with a percentage of $63.9 \%$ (53/83). At the meantime, the vast majority of these patients were treated for ventilatorassociated and healthcare-acquired pneumonia, with a percentage of $87.9 \%(73 / 83)$.

Prescribing information provided by the manufacturer suggests that tigecycline might affect the coagulation function, although an uncommon adverse reaction (incidence $<2 \%$ ) [7]. Tigecycline associated hypofibrinogenemia was not mentioned in the prescribing information. However, there are some literature reports about tigecycline associated hypofibrinogenemia in recent years [13-17], giving first place to case report [15-18], occasionally but significant. Moreover, Routsi et al. [13] in a study of 45 ICU patients on tigecycline reported that a higher dose of tigecycline treatment resulted in a decrease in fibrinogen, along with an increase in INR and APTT values, and these alterations were reversible almost immediately following tigecycline discontinuation. Zhang et al. [14] found a significantly lower fibrinogen level in patients treated with tigecycline, and the fibrinogen level returned to normal after the cessation of tigecycline. The strong time-dependent association of fibrinogen and the use of tigecycline and its reversal after discontinuation might point to a causative role of the drug.

Tigecycline was approved for administration at a loading dose of $100 \mathrm{mg}$ followed by $50 \mathrm{mg}$ twice daily [7]. However, high percentages of treatment failure and higher mortality among patients treated with the standard dose of tigecycline were documented [21-24]. Higher doses have been applied especially in patients with severe bacterial infections, but data addressing coagulation variables are limited. According to our study, in the higher-dose group, the fibrinogen level did not rebound after the cessation of tigecycline, but kept on drop, showing a more pronounced impact on fibrinogen than recommended dose. Zhang et al. [14] reported that the fibrinogen level decreased more in the higher-dose group, showing significant difference from recommended-dose group.

The use of tigecycline in patients with more severe reductions infibrinogen may put patients at an increased risk of bleeding. Our find that the new-onset bleeding and fibrinogen drop showed almost simultaneously might point to a causative role of this drug. Fibrinogen drop should not be the only reason for bleeding. Fibrinogen decreases, combined with prolongation of clotting time or/and platelet decreases, can cause severe bleeding, increased hospitalization, and mortality. Although it was not mentioned in the prescribing information, and there was a low proportion of new-onset bleeding in our study and few reports in the past, the importance of this phenomenon can't be overstated. 
Being structurally similar to tetracycline class antibiotics, tigecycline can influence coagulation either via an effect on the vitamin-K-producing flora of the gut or via a direct drug effect on the coagulation cascade [25]. Indeed, alterations of other coagulation indexes, such as prolongation of APTTLPT and INR in patients treated with tigecycline have been reported. However, decreased fibrinogen levels have not been described in the clinical trials. Whether the decreased concentration of fibrinogen was due to increased consumption or impaired synthesisis questioned. It was recommended that the cause of fibrinogen decrease should be looked for in the field of reduced synthesis.

The study reported here must be considered preliminary due to its small sample and observational characteristics. It is not routine to monitor coagulation function during tigecycline treatment in clinical works up to now, and the data we collected was with high dispersivity. The data after tigecycline withdrawal was even fewer, because these subjects were critically ill patients, many of whom were ended with death missing data after tigecycline withdrawal.

We believe that monitoring coagulation markers, including fibrinogen should be considered in all patients receiving tigecycline. If patients do develop hypofibrinogenemia or active bleeding begins, one should consider stopping tigecycline. Usually, along with the fibrinogen improvement, the coagulation function would rebound after tigecycline withdrawal. It was recommend that fibrinogen levels of $<1.2 \mathrm{~g} / \mathrm{L}$ should be treated with cryoprecipitate, or fibrinogen infusion should be given [13]. We envision further studies to clarify the mechanisms and risk factors for the observed effects.

\section{Declarations}

\section{Acknowledgments}

The authors would like to express our thanks to the staff of the IT departmrnt, Wuxi People's Hospital of Nanjing Medical University, for their technical assistance.

This research was partly supported by National Natural Science Foundation of China (No. 81401619 to Jiao-jie Hui) and Youth Medical Talent Project by

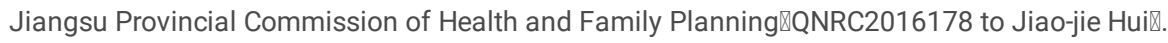

\section{Author contributions}

Junfeng Heng, Xiuhong Zhang, Qiuhui Wang and Hongyang Xucollected the data. Fengming Liang undertook the statistical analyses. Jiaojie Hui supervised the work, wrote the first draft of the manuscript, provided funding for this study. All authors reviewed the manuscript.

\section{Conflict of interests}

The authors declare no conflict of interest.

\section{References}

1. Vincent JL, Rello J, Marshall J, Silva E, Anzueto A, Martin CD, et al. International study of the prevalence and outcomes of infection in intensive care units. JAMA. 302, 2323-2329 (2009).

2. Schwaber MJ, Carmeli Y. Antimicrobial resistance and patient outcomes: the hazards of adjustment. Crit Care. 10, 164 (2006).

3. Hoffman-Roberts HL, C BE, Mitropoulos IF. Investigational new drugs for the treatment of resistant pneumococcal infections. Expert Opin Investig Drugs. 14, 973-995 (2005).

4. Hanberger $\mathrm{H}$, Antonelli M, Holmbom M, Lipman J, Pickkers $\mathrm{P}$, Leone M, et al. Infections, antibiotic treatment and mortality in patients admitted to ICUs in countries considered to have high levels of antibiotic resistance compared to those with low levels. BMC Infect Dis. 14, 513 (2014).

5. Dabar G, Harmouche C, Salameh P, Jaber BL, Jamaleddine G, Waked M, et al. Community- and healthcare-associated infections in critically ill patients: a multicenter cohort study. Int J Infect Dis. 37, 80-85 (2015).

6. Sader HS, Jones RN, Dowzicky MJ, Fritsche TR. Antimicrobial activity of tigecycline tested against nosocomial bacterial pathogens from patients hospitalized in the intensive care unit. Diagn Microbiol Infect Dis. 52, 203-208 (2005).

7. Wyeth Pharmaceuticals, Inc. Tygacil-tigecycline injection, powder, lyophilized, for solution. Wyeth Pharmaceuticals, Inc., Philadelphia, PA. 2011.

8. Guirao X, Sánchez García M, Bassetti M, Bassetti M, Bodmann KF, Dupont H, et al. Safety and tolerability of tigecycline for the treatment of complicated skin and soft-tissue and intra-abdominal infections: an analysis based on five European observational studies. J Antimicrob Chemother. 68, ii37-44 (2013).

9. Ellis-Grosse EJ, Babinchak T, Dartois N, Rose G, Loh E. The efficacy and safety of tigecycline in the treatment of skin and skin-structure infections: results of 2 double-blind phase 3 comparison studies with vancomycin-aztreonam. Clin Infect Dis. 41, S341-353 (2005).

10. Wu X, Zhao P, Dong L, Zhang X. A case report of patient with severe acute cholangitis with tigecycline treatment causing coagulopathy and hypofibrinogenemia. Medicine. 96, e9124 (2017).

11. Leng B, Xue YC, Zhang W, Gao T, Yan G, Tang H. A retrospective analysis of the effect of tigecycline on coagulation function. Chem Pharm Bull. 67, 258264 (2019).

12. Sabanis N, Paschou E, Gavriilaki E, Kalaitzoglou A, Vasileiou S. Hypofibrinogenemia induced by tigecycline: a potentially life-threatening coagulation disorder. Infect Dis. 47, 743-746 (2015).

13. Routsi C, Kokkoris S, Douka E, Ekonomidou F, Karaiskos I, Giamarellou H. High-dose tigecycline-associated alterations in coagulation parameters in critically ill patients with severe infections. Int J Antimicrob Agents. 45, 90-93 (2015). 
14. Zhang Q, Zhou S, Zhou J. Tigecycline treatment causes a decrease in fibrinogen levels. Antimicrob Agents Chemother. 59, 1650-1655 (2015) .

15. Sabanis N, Paschou E, Gavriilaki E, Kalaitzoglou A, Vasileiou S. Hypofibrinogenemia induced by tigecycline: a potentially life-threatening coagulation disorder. Infect Dis (Lond). 47, 743-746 (2015).

16. Rossitto G, Piano S, Rosi S, Simioni P, Angeli P. Life-threatening coagulopathy and hypofibrinogenaemia induced by tigecycline in a patient with advanced liver cirrhosis. Eur J Gastroenterol Hepatol. 26, 681-684 (2014).

17. Pieringer H, Schmekal B, Biesenbach G, Pohanka E. Severe coagulation disorder with hypofibrinogenemia associated with the use of tigecycline. Ann Hematol. 89, 1063-1064 (2010).

18. Balfousias T, Apostolopoulos AP, Angelis S, Maris S, Papanikolaou A. Spontaneous knee hemarthrosis due to hypofibrinogenemia following tigecycline treatment for periprosthetic joint infection. Cureus. 11, e5883 (2019).

19. Magiorakos AP, Srinivasan A, Carey RB, Carmeli Y, Falagas ME, Giske CG, et al. Multidrug-resistant, extensively drug-resistant and pandrug-resistant bacteria: an international expert proposal for interim standard definitions for acquired resistance. Clin Microbiol Infect. 18, 268-281 (2012).

20. Kwa AL, Low JG, Lee E, Kurup A, Chee HL, Tam VH. The impact of multidrug resistance on the outcomes of critically ill patients with Gram-negative bacterial pneumonia. Diagn Microbiol Infect Dis. 58, 99-104 (2007).

21. Vardakas KZ, Rafailidis PI, Falagas ME. Effectiveness and safety of tigecycline: focus on use for approved indications. Clin Infect Dis. 54, $1672-1674$ (2012).

22. Prasad P, Sun J, Danner RL, Natanson C. Excess deaths associated with tigecycline after approval based on noninferiority trials. Clin Infect Dis. 54, $1699-$ 1709 (2012).

23. Tasina E, Haidich AB, Kokkali S, Arvanitidou M. Efficacy and safety of tigecycline for the treatment of infectious diseases: a meta-analysis. Lancet Infect Dis. 11, 834-844 (2011).

24. Yahav D, Lador A, Paul M, Leibovici L. Efficacy and safety of tigecycline: a systematic review and meta-analysis. J Antimicrob Chemother. 66, 1963-1971 (2011).

25. Doan TL, Fung HB, Mehta D, Riska PF. Tigecycline: a glycylcycline antimicrobial agent. Clin Ther. 28, 1079-1106 (2006).

\section{Figures}

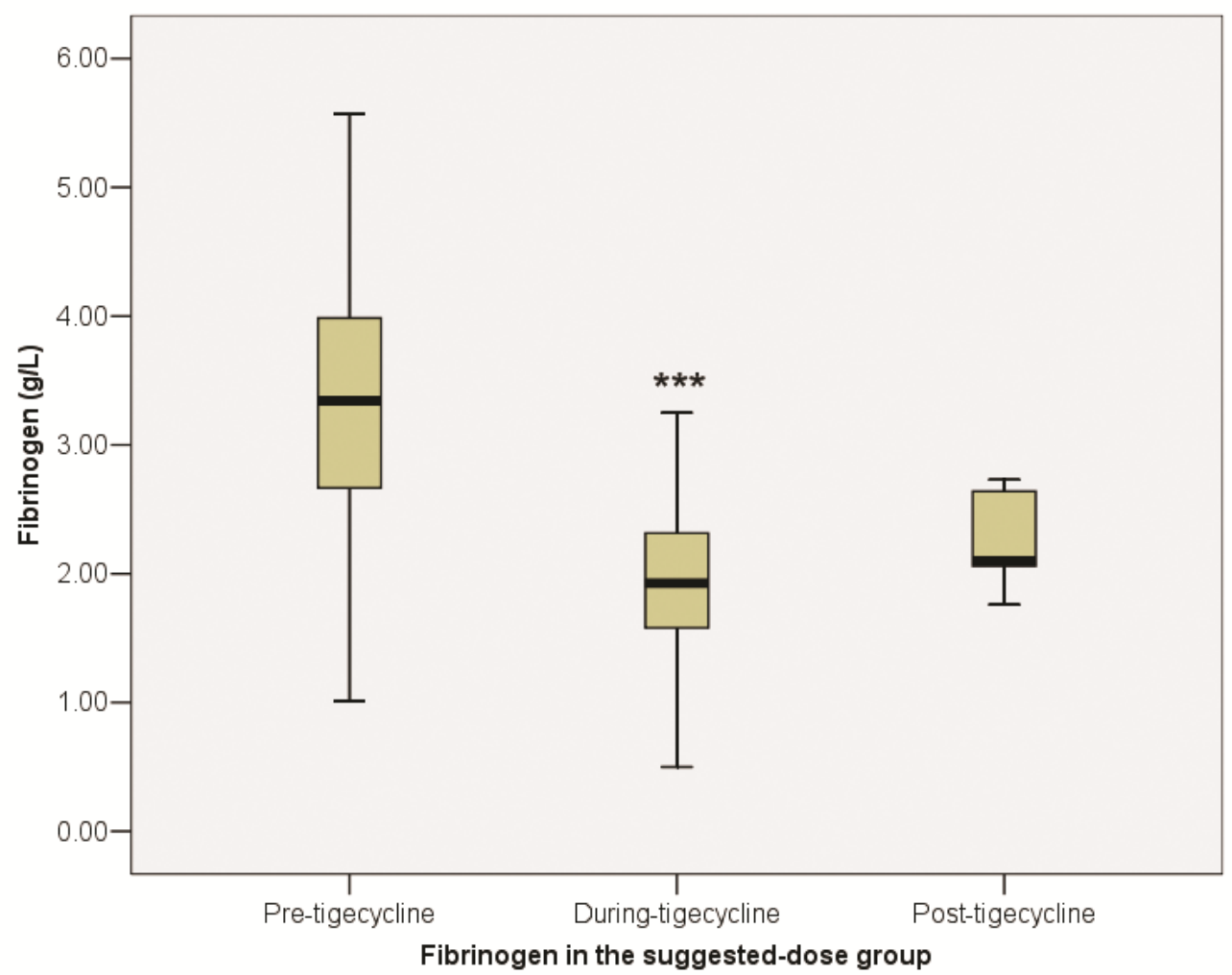

Figure 1

The fibrinogen level dropped significantly during tigecycline treatment in the suggested-dose group and rebounded after the cessation of treatment. $\star \star \star$, compared with the level of before tigecycline treatment, $P=0.000$. 


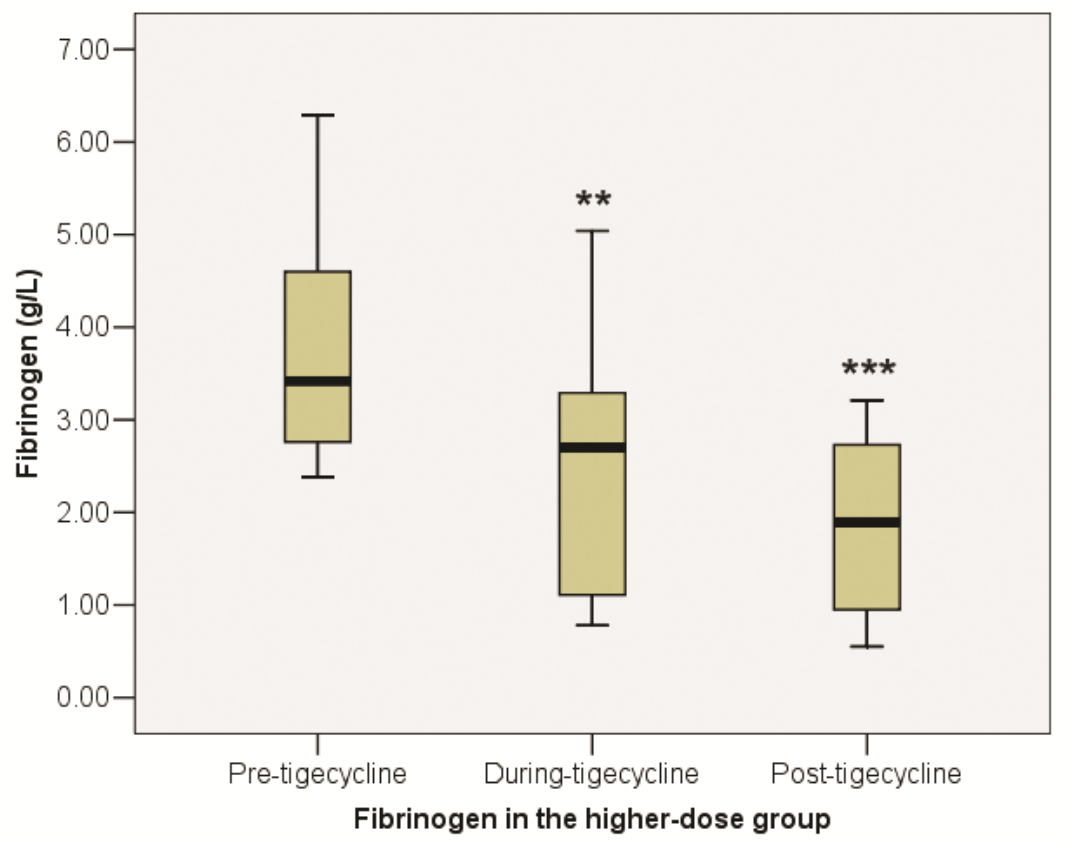

Figure 2

The fibrinogen level dropped significantly during tigecycline treatment in the higher-dose group and kept on drop after the cessation of treatment. **, compared with the level of before tigecycline treatment, $P=0.009 ; * \star \star$, compared with the level of before tigecycline treatment, $P=0.000$. 